\title{
Enhanced vortex heat conductance in mesoscopic superconductors
}

\author{
N. B. Kopnin ${ }^{(1,2)}$, A. S. Mel'nikov ${ }^{(3)}$, V. I. Pozdnyakova ${ }^{(3)}$, \\ D. A. Ryzhov (3), I. A. Shereshevskii (3), and V. M. Vinokur (4) \\ (1) Low Temperature Laboratory, Helsinki University of Technology, P.O. Box 2200, FIN-02015 HUT, Finland, \\ (2) L. D. Landau Institute for Theoretical Physics RAS, 117940 Moscow, Russia, \\ (3) Institute for Physics of Microstructures RAS, 603950, Nizhny Novgorod, GSP-105, Russia, \\ (4) Argonne National Laboratory, Argonne, Illinois 60439
}

(Dated: January 25, 2020)

\begin{abstract}
Electronic heat transport along the flux lines in a long ballistic mesoscopic superconductor cylinder with a radius of the order of several coherence lengths is investigated theoretically using both semiclassical approach and the full quantum-mechanical analysis of the Bogoliubov-de Gennes equations. The semiclassical approach is constructed analogously to the Landauer transport theory in mesoscopic conductors employing the idea that heat is carried by the quasiparticle modes propagating along the vortex core. We show that the vortex heat conductance in a mesoscopic sample is strongly enhanced as compared to its value for a bulk superconductor; it grows as the cylinder radius decreases. This unusual behavior results from a strongly increased number of single-particle transport modes due to giant mesoscopic oscillations of energy levels, which originate from the interplay between the Andreev reflection at the vortex core boundary and the normal reflection at the sample edge. We derive the exact quantum-mechanical expression for the heat conductance and solve the Bogoliubov-de Gennes equations numerically. The results of numerical computations confirm the qualitative Landauer-type picture and allow us to take into account the partial reflections of excitations. We analyze the effect of surface imperfections on the spectrum of core excitations. We show that the giant oscillations of core levels and thus the essential features of the heat transport characteristic to ideal mesoscopic samples hold for a broad class of surface imperfections as well.
\end{abstract}

PACS numbers: 74.78.Na, 74.25.Fy, 74.25.Op, 74.45.+c

\section{INTRODUCTION}

Low-temperature measurements of the heat transport in the mixed state of type-II superconductors is a unique tool providing information about characteristics of the quasiparticle excitations and superconducting gap structure. When the external magnetic field $B$ is applied, thermal transport in the direction of field is dominated by the quasiparticle waves propagating along the vortex cores. Thermal conductivity in the mixed state is a longstanding problem considered in many classical papers (see, e.g., Refs. [1 2] where the situation near the upper critical field $H_{c 2}$ was discussed). In dirty superconductors, the electron contribution to the thermal conductance along the vortices is proportional to the area occupied by the cores $\kappa(B) \simeq\left(B / H_{c 2}\right) \kappa_{N}$, where $\kappa_{N}$ is the electron thermal conductance in the normal state 3.4 .5 In clean superconductors this simple estimate fails to describe the experimental data 6.7 : the thermal conductance along the well-separated vortices in $s$-wave superconductors appears to be two orders of magnitude smaller than predicted. This discrepancy was first explained in Ref. 7] as resulting from the very small group velocity of the Caroli-de Gennes-Matricon (CdGM) states ${ }^{8}$ localized inside the cores. Ideas of Ref. [7] were cast into a quantitative theoretical framework in Ref. [9] where the vortex line was treated as a long ballistic quantum channel. It was shown that the electronic thermal transport through a vortex can be described within the framework of the Landauer approach in a manner similar to that used for heat transport in normal conductors ${ }^{10.11}$. The vortex heat conductance can be expressed in terms of the "heat conductance quanta" as a certain amount of heat carried by each quantized conducting mode $\frac{12}{2}$. This yields $\kappa_{\mathrm{L}}=\kappa_{0} N_{\mathrm{L}}$, where the quantum $\kappa_{0}=\pi T /(3 \hbar)$ is the universal heat conductance per conducting mode in a normal metal (we use the units with $k_{B}=1$ ), and $N_{\mathrm{L}}$ is the effective number of conducting modes in the vortex (for both spin projections of electron). Quantization of the heat conductance in normal conductors has been observed in several experiments (see, e.g., Refs. 11 13]).

To estimate the number of modes $N_{\mathrm{L}}$ we summarize the main properties of the vortex core spectrum. The CdGM spectrum, $\epsilon_{\mu}$, is a function of the quantized (halfinteger) angular momentum $\mu=b k_{\perp}$, where $b$ is the impact parameter, $k_{\perp}=\sqrt{k_{F}^{2}-k_{z}^{2}}, k_{F}$ is the Fermi momentum, and $k_{z}$ is the momentum projection on the vortex axis. As $\mu$ changes from $-\infty$ to $+\infty, \epsilon_{\mu}$ varies from $-\Delta_{0}$ to $+\Delta_{0}$ crossing zero when $\mu$ changes its sign. Here $\Delta_{0}$ is the gap value far from the vortex axis. At small energies, $|\varepsilon| \ll \Delta_{0}$, the spectrum is $\epsilon_{\mu} \simeq-\mu \Delta_{0} /\left(k_{\perp} \xi\right)$, where $\xi=\hbar V_{F} / \Delta_{0}$ is the superconducting coherence length, and $V_{F}=\hbar k_{F} / m$ is the Fermi velocity. The interlevel spacing $\omega_{0} \simeq \Delta_{0} /\left(k_{F} \xi\right)$ taken at $k_{z}=0$ determines the excitation minigap $\omega_{0} / 2$. For temperatures $\omega_{0} \ll T \ll \Delta_{0}$, the number of modes is thus ${ }^{9} N_{\mathrm{L}} \sim T / \omega_{0}$, and the heat conductance of one vortex becomes

$$
\kappa_{\mathrm{L}} \sim T^{2} / \hbar \omega_{0}
$$

This estimate can also be obtained using the Sharvin conductance with the group velocity $v_{g}=\partial \epsilon_{\mu} / \hbar \partial k_{z} \sim$ $\epsilon_{\mu} / \hbar k_{F}$ rather than $v_{z} \sim v_{F}$ as in a normal tube. Com- 
paring $N_{\mathbf{L}}$ with the corresponding Sharvin's number of channels in the normal wire of radius $\xi, N_{\mathrm{Sh}} \sim\left(k_{F} \xi\right)^{2}$, one sees that $N_{\mathrm{L}} / N_{\mathrm{Sh}} \sim v_{g} / v_{F} \sim\left(T / T_{c}\right)\left(k_{F} \xi\right)^{-1} \ll 1$. At extremely low temperatures the minigap in the spectrum suppresses the heat transport exponentially: $\kappa \propto$ $\exp \left(-\omega_{0} / 2 T\right)$ when $T \ll \omega_{0}$.

The qualitative estimate of Eq. (11) applies to the heat conductance along the vortex core in the samples infinite in the lateral direction, but as we will see below does not generally work for samples finite in the lateral direction. Recent remarkable progress in experimental technique made it possible direct heat transport measurements in mesoscopic normal-superconducting heterostructures ${ }^{14}$ of sizes comparable to the superconducting coherence length, opening, in particular a new route for the analysis of quantum heat transport through individual vortices in mesoscopic superconductors whose exotic vortex states are in the focus of current research ${ }^{15}$. This calls for a comprehensive quantitative theory of the heat transport in mesoscopic superconductors.

In this paper we develop a theory for an electronic heat conductance along the normal channels (vortex cores) in the superconductors of the arbitrary lateral size and shape, focusing on the specific features that appear in the mesoscopic cylinders with diameters comparable to the coherence length $\xi$. We show that in finite superconductors, especially those with the a transverse size of the order of a few coherence lengths $\xi$, the estimate Eq. (1) is no longer valid. The quantum mechanics of quasiparticle excitations in mesoscopic superconductors turns out to be very sensitive to the boundary effects. In particular, the interplay between the Andreev reflection at the core boundary and the normal scattering of quasiparticles at the sample surface results in an oscillatory behavior of the energy levels as functions of the Fermi momentum and the sample lateral size ${ }^{16}$. These conclusions have been recently confirmed also for a graphene channel in contact with a superconducting environment $\frac{17}{}$. For a vortex in a mesoscopic sample, the amplitudes of level oscillations can well exceed the CdGM interlevel spacing. In this case, the new quasiparticle modes propagating along the vortex core appear which leads to a dramatic increase in the effective number of the conducting channels, $N_{\mathrm{L}}$, as compared to that in Eq. (1). We present a theoretical analysis of the single-particle transport mechanisms in the mixed state of mesoscopic superconductors taking into account normal reflections of the quasiparticle waves at the superconductor boundaries. We concentrate on the low-temperature $T \ll \Delta_{0}$ regime for the ballistic samples with the mean free paths, both elastic and inelastic, being much larger than the sample dimensions. We further investigate the effects of the surface roughness on the excitation spectrum and, accordingly, on the heat transport. We show that the significant increase in the number of conducting modes caused by the spectrum oscillations is a generic feature of a broad class of surface imperfections.

The paper is organized as follows. In Section II we introduce the model and consider the basic equations describing the quasiparticle quantum mechanics for a vortex line placed in a mesoscopic superconducting cylinder with the surface of an arbitrary shape. A Landauer-type analysis of the heat transport due to the quasiparticle modes propagating along the vortex core is carried out in the Section III In this section we also present the results of numerical calculations of the vortex heat conductance in an ideal mesocopic cylinder. Then in the Section IV we investigate the effect of the surface roughness on the quasiparticle spectrum.

\section{QUASIPARTICLE SPECTRUM FOR A VORTEX LINE IN A MESOSCOPIC SUPERCONDUCTOR}

\section{A. Semiclassical equations}

In this section we consider a vortex placed in a superconducting mesoscopic cylinder with an arbitrary $(x, y)$ cross section and derive an eikonal approximation of the Bogoliubov-de Gennes (BdG) equation that allows us to obtain the vortex-core states in such samples. Let the vortex line be parallel to the cylinder axis $z$. We choose the origin of the polar coordinate system $(r, \theta, z)$ of the $(x y)$ plane at the vortex center. The surface of the sample is then defined by the equation $r=R(\theta)$. Since the momentum component along the vortex axis is a conserved quantity, we choose the particle- and hole-like parts of the wave function as $(U, V)=(u, v) \exp \left(i k_{z} z\right)$ respectively. The $\mathrm{BdG}$ equations have the form

$$
\left(\begin{array}{cc}
\hat{H}_{0}(\hat{\mathbf{p}}) & \Delta(x, y) \\
\Delta^{*}(x, y) & -\hat{H}_{0}^{*}(\hat{\mathbf{p}})
\end{array}\right)\left(\begin{array}{l}
u \\
v
\end{array}\right)=\varepsilon\left(\begin{array}{l}
u \\
v
\end{array}\right) .
$$

Here

$$
\hat{H}_{0}(\hat{\mathbf{p}})=\frac{1}{2 m}\left(\hat{\mathbf{p}}-\frac{e}{c} \mathbf{A}\right)^{2}-E_{\perp},
$$

$\hat{\mathbf{p}}=-i \hbar \nabla, E_{\perp}=E_{F}-\hbar^{2} k_{z}^{2} /(2 m)=\hbar^{2} k_{\perp}^{2} /(2 m)$, and $E_{F}$ is the Fermi energy. The order parameter profile $\Delta(x, y)$ is homogeneous in the $z$ direction,

$$
\Delta(x, y)=\Delta_{0} \delta_{v}(r) e^{i \varphi+i \tilde{\varphi}} .
$$

Here $\delta_{v}(r)$ is the normalized order parameter magnitude for a vortex centered at $r=0$, such that $\delta_{v}(r)=1$ for $r \rightarrow \infty, e^{i \varphi}=(x+i y) / r$ is the vortex phase factor, and $\tilde{\varphi}$ is the part of the order parameter phase induced by the boundary effects; $\tilde{\varphi}$ having no singularities inside the superconductor. For simplicity we start our analysis with the case of the weak external magnetic field and the extreme type-II superconductors where the vector potential A can be neglected. The effects of the magnetic field are discussed later in Section

Let us introduce a momentum representation:

$$
\left(\begin{array}{l}
u \\
v
\end{array}\right)=\frac{1}{(2 \pi \hbar)^{2}} \int d^{2} p e^{i \mathbf{p r} / \hbar} \hat{\Psi}(\mathbf{p})
$$


where $\mathbf{p}=p\left(\cos \theta_{p}, \sin \theta_{p}\right)=p \mathbf{p}_{0}$. The unit vector $\mathbf{p}_{0}$ parametrized by the angle $\theta_{p}$ defines the trajectory direction in the $(x, y)$ plane. Within the quasiclassical approach the wave function in the momentum representation assumes the form:

$$
\hat{\Psi}(\mathbf{p})=\frac{1}{k_{\perp}} \int_{-\infty}^{+\infty} d s e^{-i\left(|\mathbf{p}|-\hbar k_{\perp}\right) s / \hbar} \hat{\psi}\left(s, \theta_{p}\right) .
$$

We look for solutions with $|\mathbf{p}| \approx \hbar k_{\perp}$. For the slowly varying part of the wavefunction $\hat{\psi}$ we obtain the quasiclassical (Andreev) equation along a trajectory specified by an orientation angle, $\theta_{p}$, and an impact parameter, $b$, with respect to the vortex center. The distance along the trajectory is $s=r \cos \left(\theta_{p}-\theta\right)$. The wavefunction for $\mathbf{r}=r(\cos \theta, \sin \theta)$ is derived from Eqs. (3), (4):

$$
\left(\begin{array}{l}
u \\
v
\end{array}\right)=\frac{1}{2 \pi} \int_{0}^{2 \pi} d \theta_{p} e^{i k_{\perp} r \cos \left(\theta_{p}-\theta\right)} \hat{\psi}\left(r \cos \left(\theta_{p}-\theta\right), \theta_{p}\right) .
$$

The wave functions should vanish at the sample surface:

$$
\frac{1}{2 \pi} \int_{0}^{2 \pi} d \theta_{p} e^{i k_{\perp} R(\theta) \cos \left(\theta_{p}-\theta\right)} \hat{\psi}\left(R(\theta) \cos \left(\theta_{p}-\theta\right), \theta_{p}\right)=0 .
$$

We introduce the angular-momentum expansion

$$
\hat{\psi}=\sum_{\mu} e^{i \mu \theta_{p}+i \hat{\sigma}_{z}\left[\theta_{p}+\tilde{\varphi}\left(s, \theta_{p}\right)\right] / 2} \hat{g}_{\mu}\left(s, \theta_{p}\right),
$$

where $\mu=-k_{\perp} b=n+1 / 2$, and $n$ is an integer. The function $\hat{g}_{\mu}$ satisfies the equation: $\hat{H} \hat{g}_{\mu}=\varepsilon \hat{g}_{\mu}$, where

$$
\begin{aligned}
\hat{H}= & -i \hbar V_{\perp} \hat{\sigma}_{z} \frac{\partial}{\partial s}+\Delta_{0} \delta_{v}(r)\left[\hat{\sigma}_{x} s-\hat{\sigma}_{y} b\right] / r \\
& +\hbar k_{\perp} \tilde{v}_{s}\left(s, b, \theta_{p}\right)
\end{aligned}
$$

$V_{\perp}=\hbar k_{\perp} / m, \hat{\sigma}_{x}, \hat{\sigma}_{y}, \hat{\sigma}_{z}$ are the Pauli matrices, $r=$ $\sqrt{s^{2}+b^{2}}$, and

$$
\tilde{v}_{s}=\frac{\hbar}{2 m} \frac{\partial \tilde{\varphi}}{\partial s}=\frac{\hbar}{2 m} \mathbf{p}_{0} \cdot \nabla \tilde{\varphi}
$$

is the projection on the momentum direction of the superfluid velocity component induced by the boundary effects; we neglect this velocity assuming for simplicity that it is small as compared to the critical velocity at distances shorter than $\xi$ from the vortex axis. The function $\hat{g}_{\mu}$ is thus independent of $\theta_{p}: \hat{g}_{\mu}=\hat{g}_{\mu}(s)$. This assumption does not change the main results of our work.

The main contributions to the integral in Eq. (6) come from the vicinities of the stationary phase points, $\sin \left(\theta_{p}-\right.$ $\theta)=\mu / k_{\perp} R(\theta)$. We further assume that the r.h.s. of this equation is small, i.e. that the impact parameters for all trajectories contributing to the solution are much less than the system size. As a result we obtain two stationary points: $\theta_{p} \simeq \theta+\mu / k_{\perp} R(\theta)$ and $\theta_{p} \simeq \pi+$ $\theta-\mu / k_{\perp} R(\theta)$. Furthermore, we neglect the corrections $\mu / k_{\perp} R$ in the above expressions for stationary angles; this is valid provided (i) the additional phase shifts along trajectories caused by the finite impact parameters are small $k_{\perp} b^{2} / R \ll 1$, and (ii) $\hat{g}_{\mu}(s)$ is a slow function on the atomic length scale.

Near the stationary angle we can write $\cos \left(\theta_{p}-\theta\right) \simeq$ $1-\left(\theta_{p}-\theta\right)^{2} / 2$ and $\cos \left(\theta_{p}-\theta\right) \simeq-1+\left(\theta_{p}-\theta-\pi\right)^{2} / 2$, respectively. The integral in Eq. (6) becomes

$$
\begin{aligned}
& \int_{0}^{2 \pi} d \theta_{p} e^{i k_{\perp} R(\theta) \cos \left(\theta_{p}-\theta\right)} \hat{\psi}\left[R(\theta) \cos \left(\theta_{p}-\theta\right), \theta_{p}\right] \\
= & \sqrt{\frac{2 \pi}{k_{\perp} R(\theta)}\left[e^{i k_{\perp} R(\theta)-i \pi / 4} \hat{\psi}(R(\theta), \theta)\right.} \\
& \left.+e^{-i k_{\perp} R(\theta)+i \pi / 4} \hat{\psi}(-R(\theta), \theta+\pi)\right] .
\end{aligned}
$$

Thus, the boundary condition Eq. (6) takes the form:

$$
\hat{\psi}(R(\theta), \theta)=-e^{-2 i k_{\perp} R(\theta)+i \pi / 2} \hat{\psi}(-R(\theta), \theta+\pi) .
$$

To find the function $\hat{g}_{\mu}$ we can use the results of Ref. 9 . Introducing

$$
\hat{g}_{\mu}=e^{\hat{\sigma}_{z}\left(i \frac{\pi}{2}-i \frac{\pi}{4} \operatorname{sign} b-\frac{i}{2} \arctan \frac{s}{b}\right)} \hat{w}_{\mu}
$$

we obtain:

$$
-i \hbar V_{\perp} \hat{\sigma}_{z} \frac{\partial}{\partial s} \hat{w}_{\mu}+\hat{\sigma}_{x} \Delta_{0} \delta_{v} \hat{w}_{\mu}=\left(\varepsilon+\frac{\hbar V_{\perp}}{2} \frac{b}{s^{2}+b^{2}}\right) \hat{w}_{\mu} .
$$

The two linearly independent solutions for low energies are $\stackrel{9}{=}$

$$
\begin{aligned}
& \hat{w}_{1 \mu}(s)=e^{-D(s) / 2} e^{\frac{i}{2} \arctan (s / b) \hat{\sigma}_{z}+i \hat{\sigma}_{z}[\operatorname{sign}(b)-1] \pi / 4} \\
& \times \sqrt{\frac{e^{D-|D|}+\gamma^{2}}{\left(1+\gamma^{2}\right)\left(e^{2 D}+\gamma^{2}\right)}}\left[e^{D}+i \gamma \hat{\sigma}_{z}\right] \hat{\lambda},
\end{aligned}
$$

and $\hat{w}_{2 \mu}(s)=\operatorname{sign}(b) \hat{w}_{1 \mu}^{*}(-s)$. We denote

$$
\hat{\lambda}=\left(\begin{array}{l}
1 \\
1
\end{array}\right)
$$

$$
\begin{gathered}
D(s)=\frac{2 \Delta_{0}}{\hbar V_{\perp}} \int_{0}^{s} \delta_{v}\left(s^{\prime}\right) d s^{\prime}, \Lambda=\frac{2 \Delta_{0}}{\hbar V_{\perp}} \int_{0}^{\infty} e^{-D(s)} d s \\
\gamma(\mu)=\frac{2}{\hbar V_{\perp}}[\epsilon(\mu)-\varepsilon] \int_{0}^{\infty} e^{-D(s)} d s .
\end{gathered}
$$

Here

$$
\epsilon(\mu)=-\frac{2 \Delta_{0}^{2} \mu}{\hbar k_{\perp} V_{\perp} \Lambda} \int_{0}^{\infty} \frac{\delta(s)}{s} e^{-D(s)} d s \simeq-\frac{\mu \omega_{0} k_{F}}{k_{\perp}}
$$


is the CdGM energy spectrum for an infinite sample.

We take the function $\hat{g}_{\mu}$ as a sum

$$
\hat{g}_{\mu}=c_{1 \mu} \hat{G}_{1 \mu}+c_{2 \mu} \hat{G}_{2 \mu}
$$

of two linearly independent solutions

$$
\begin{aligned}
& \hat{G}_{1 \mu}=e^{+\hat{\sigma}_{z}\left(i \frac{\pi}{2}-i \frac{\pi}{4} \operatorname{sign} b-\frac{i}{2} \arctan \frac{s}{b}\right)}\left(\hat{w}_{1 \mu}+\hat{w}_{2 \mu}\right) / 2, \\
& \hat{G}_{2 \mu}=e^{+\hat{\sigma}_{z}\left(i \frac{\pi}{2}-i \frac{\pi}{4} \operatorname{sign} b-\frac{i}{2} \arctan \frac{s}{b}\right)}\left(\hat{w}_{1 \mu}-\hat{w}_{2 \mu}\right) / i \gamma .
\end{aligned}
$$

The characteristic length scale of the function $\hat{g}_{\mu}$ is of the order of $\xi$. Assuming $\gamma \ll 1$ these two solutions can be rewritten in the form:

$$
\begin{aligned}
& \hat{G}_{1 \mu}=e^{i \hat{\sigma}_{z} \pi / 4}\left(e^{-|D(s)| / 2}-i \operatorname{sign} s \frac{\gamma}{2} \hat{\sigma}_{z} e^{|D(s)| / 2}\right) \hat{\lambda} \\
& \hat{G}_{2 \mu}=e^{i \hat{\sigma}_{z} \pi / 4} e^{|D(s)| / 2} \hat{\sigma}_{z} \hat{\lambda} .
\end{aligned}
$$

Substituting all the above expressions into the boundary condition and taking account of the fact that for small $\mu$ we have $\tilde{\varphi}(R, \theta) \simeq \tilde{\varphi}(-R, \theta+\pi)$, we obtain

$$
\begin{aligned}
& \sum_{\mu} e^{i \mu \theta}\left\{c_{1 \mu}\left(e^{-D_{\theta} / 2}-i \frac{\gamma}{2} \hat{\sigma}_{z} e^{D_{\theta} / 2}\right)+c_{2 \mu} e^{D_{\theta} / 2} \hat{\sigma}_{z}-\right. \\
& \left.-e^{i \pi \mu-i \alpha_{\theta}}\left[c_{1 \mu}\left(\hat{\sigma}_{z} e^{-D_{\theta} / 2}+i \frac{\gamma}{2} e^{D_{\theta} / 2}\right)+c_{2 \mu} e^{D_{\theta} / 2}\right]\right\} \hat{\lambda}=0
\end{aligned}
$$

where $\alpha_{\theta}=2 k_{\perp} R(\theta), D_{\theta}=D(R(\theta))$. These expressions hold as long as $e^{-D_{\theta}} \ll 1$. Let us now introduce the angular functions

$$
c_{j}(\theta)=\sum_{\mu} e^{i \mu \theta} c_{j \mu}, c_{j \mu}=\frac{1}{2 \pi} \int_{0}^{2 \pi} e^{-i \mu \theta} c_{j}(\theta) d \theta .
$$

They satisfy

$$
\begin{aligned}
-\frac{i}{2} \hat{\gamma} c_{1}(\theta)+c_{2}(\theta) & =e^{-D_{\theta}-i \alpha_{\theta}} c_{1}(\theta+\pi), \\
\frac{i}{2} \hat{\gamma} c_{1}(\theta+\pi)+c_{2}(\theta+\pi) & =e^{-D_{\theta}+i \alpha_{\theta}} c_{1}(\theta) .
\end{aligned}
$$

$\gamma$ is now considered as a function of $\mu$ which is transformed into an operator

$$
\hat{\gamma} \equiv \hat{\gamma}(\hat{\mu})=\gamma\left(-i \frac{\partial}{\partial \theta}\right)
$$

Since $c_{j}(\theta+2 \pi)=-c_{j}(\theta)$ while $\alpha_{\theta}$ and $D_{\theta}$ are $2 \pi$ periodic we obtain

$$
\begin{aligned}
c_{2}(\theta+\pi) & =\frac{1}{2}\left(e^{-D_{\theta}+i \alpha_{\theta}}-e^{-D_{\theta+\pi}-i \alpha_{\theta+\pi}}\right) c_{1}(\theta), \\
i \hat{\gamma} c_{1}(\theta+\pi) & =\left(e^{-D_{\theta}+i \alpha_{\theta}}+e^{-D_{\theta+\pi}-i \alpha_{\theta+\pi}}\right) c_{1}(\theta) .
\end{aligned}
$$

This set of equations demonstrates that the normal reflection at the surface couples the two trajectories going in the opposite directions that are described by two independent functions $c_{1}(\theta)$ and $c_{1}(\theta+\pi)$. Our derivation uses trajectories with small impact parameters and, thus, assumes that these equations are valid provided the angular harmonics $c_{1 \mu}$ vanish for $|\mu|>\min \left\{k_{\perp} \xi, \sqrt{k_{\perp} R}\right\}$.

Following Ref. 18] it is convenient to introduce a twocomponent function

$$
\hat{c}(\theta)=\left(\begin{array}{c}
c_{1}(\theta) \\
c_{1}(\theta+\pi)
\end{array}\right),
$$

defined for $0<\theta<\pi$ with the boundary condition $\hat{c}(\theta+$ $\pi)=i \hat{\sigma}_{y} \hat{c}(\theta)$. The final equation for $\hat{c}$ reads

$$
\hat{h} \hat{c}=\varepsilon \hat{c}
$$

where the effective Hamiltonian is $\hat{h}=\hat{h}_{0}+\hat{V}$,

$$
\hat{h}_{0}=\epsilon\left(-i \frac{\partial}{\partial \theta}\right), \hat{V}=-\Lambda^{-1} \Delta_{0}\left(\begin{array}{cc}
0 & v_{\theta} \\
v_{\theta}^{*} & 0
\end{array}\right)
$$

and

$$
v_{\theta}=i\left(e^{-D_{\theta}-i \alpha_{\theta}}+e^{-D_{\theta+\pi}+i \alpha_{\theta+\pi}}\right) .
$$

Equations (15)-(17) are the central result of this Section. They provide a semiclassical description of vortex core excitations taking into account the normal scattering processes at the sample surface of a general shape. The normal scattering is introduced by the potential $\hat{V}$ through the factors $e^{i \alpha_{\theta}}$ which result in mesoscopic oscillations of the spectrum as functions of momentum and sample size ${ }^{16}$. We employ these equations to study the effects of surface imperfections further in Section IV

\section{B. Spectrum of an ideal cylinder}

For an ideal cylinder with a constant radius $R(\theta)=R$, the solution of equations (15)-(17) were obtained in Ref. [16]; we reproduce them here for the sake of completeness and convenience. The wavefunction is

$$
\hat{c}_{\mu}=\frac{e^{i \mu \theta}}{\sqrt{2 \pi}}\left(\begin{array}{c}
1 \\
e^{i \pi \mu}
\end{array}\right)
$$

where $\mu=n+1 / 2$, which gives the energy spectrum ${ }^{16}$

$$
\varepsilon_{\mu}=\epsilon(\mu)+\Delta_{0} \frac{2 e^{-D(R)}}{\Lambda} \cos \left(2 k_{\perp} R-\pi \mu+\pi / 2\right),
$$

Using a simple vortex core mode ${ }^{16}$

$$
\delta_{v}(r)=r / \sqrt{r^{2}+\xi_{v}^{2}},
$$

where $\xi_{v} \sim \xi$ is the size of the vortex core, one obtains

$$
\begin{aligned}
\frac{\varepsilon_{\mu}}{\Delta_{0}}= & -\frac{\mu}{k_{\perp} \xi_{v}} \frac{\mathcal{K}_{0}(a)}{\mathcal{K}_{1}(a)} \\
& +\frac{2 e^{-a \sqrt{R^{2} / \xi_{v}^{2}+1}}}{a \mathcal{K}_{1}(a)} \cos \left[2 k_{\perp} R-\pi \mu+\frac{\pi}{2}\right]
\end{aligned}
$$




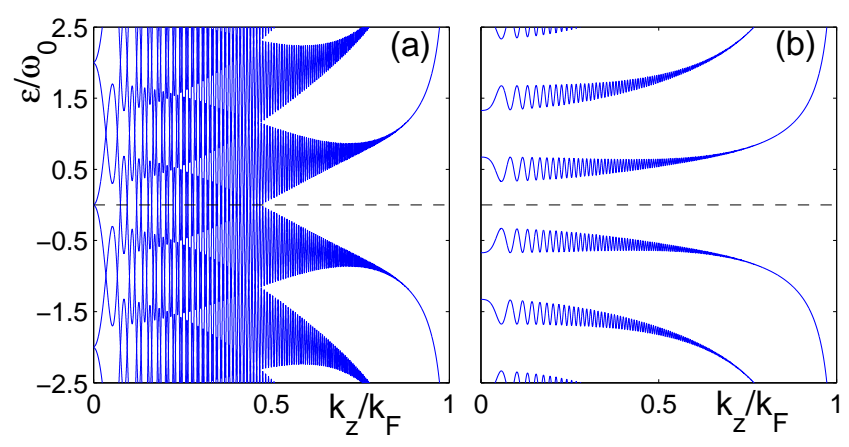

FIG. 1: The spectra of quasiparticles for a vortex in the cylinder with $R / \xi=3.5$ (a) and $R / \xi=4.5$ (b). The gap is approximated by Eq. (20) with $\xi_{v}=\xi, k_{F} \xi=200$.

Here $a=2\left(\xi_{v} / \xi\right) k_{F} / k_{\perp}$ and $\mathcal{K}_{n}$ is the McDonald function. Typical energy spectra of Eq. (21) for a vortex in a mesoscopic cylinder are shown in Fig. The minigap in the spectrum exists only for $R>R_{c}$, where the critical radius is

$$
R_{c}=\frac{\xi}{2} \sqrt{\ln ^{2}\left[\frac{2 k_{F} \xi}{\mathcal{K}_{0}\left(2 \xi_{v} / \xi\right)}\right]-\frac{4 \xi_{v}^{2}}{\xi^{2}}} \simeq \frac{\xi}{2} \ln \left[\frac{2 k_{F} \xi}{\mathcal{K}_{0}\left(2 \xi_{v} / \xi\right)}\right] .
$$

The interlevel spacing $\omega_{0}$ for CdGM states is

$$
\omega_{0}=\frac{\Delta_{0}}{k_{F} \xi_{v}} \frac{\mathcal{K}_{0}\left(2 \xi_{v} / \xi\right)}{\mathcal{K}_{1}\left(2 \xi_{v} / \xi\right)} .
$$

Taking for simplicity $\xi_{v}=\xi$ we get $\omega_{0} \simeq 0.8143 \Delta_{0} / k_{F} \xi$.

\section{Magnetic-field tuning of the spectrum oscillations}

In this section we consider the influence of an external magnetic field on the spectral characteristics and show that the quasiparticle spectrum can be tuned by changing the magnetic field $\mathbf{H}=H \mathbf{z}_{0}$ applied to the sample.

With the account of the magnetic field, the CdGM energy is renormalized ${ }^{19}, \varepsilon_{\mu} \rightarrow \varepsilon_{\mu}+\mu \omega_{c} / 2$ where $\omega_{c}=$ $e H / m c$ is the cyclotron frequency. However, the most important is the effect of the magnetic field on the gap profile. Far from the core it becomes

$$
\delta_{v} \simeq 1-\frac{\pi^{2} \xi^{2} H^{2} r^{2}}{2 \phi_{0}^{2}} .
$$

where $\phi_{0}=\pi \hbar c / e$ is the magnetic flux quantum. We assume that the supercurrent density at the sample edge is less than the critical current so that the role of states bound to the edge $e^{20}$ can be neglected for low energies. The change in the gap profile results in an additional small renormalization of the minigap and, what is more important, it also affects the amplitude of level oscillations determined by the factor $\exp (-D(R))$. Indeed,we find from Eqs. (11), (24)

$$
D(R)=\frac{k_{F}}{k_{\perp}}\left(\frac{2 R}{\xi}-\frac{\xi h^{2}}{3 R}\right)
$$

where $h=H / H_{0}$ and $H_{0}=\phi_{0} / \pi R^{2}$. The field $H_{0}$ is of the order of the critical field $H_{1}=\left(\phi_{0} / \pi R^{2}\right) \ln (R / \xi)$ at which the vortex becomes energetically favorable in the sample. The ratio of the oscillation amplitudes taken, e.g., for $h=3$ and $h=1$ is given by the expression:

$$
\frac{e^{-D(R, h=3)}}{e^{-D(R, h=1)}}=\exp \left(\frac{8 k_{F} \xi}{3 k_{\perp} R}\right) .
$$

For $k_{\perp}=k_{F}$ and $R / \xi=4$ this estimate gives us a factor $\sim 2$, which shows that the amplitude of oscillations can be varied significantly by changing the magnetic field in a reasonable range. The critical radius $R_{c}$ at which zero energy modes appear is determined by the condition $D\left(R_{c}, H\right) \simeq \ln \left(k_{F} \xi\right)$ and does also depend on $H$. To conclude here, one observes that the energy level oscillations and the number of propagating quasiparticle modes depend strongly on the external magnetic field and thus can be effectively controlled in the experiments.

\section{THE LANDAUER APPROACH TO THE VORTEX HEAT CONDUCTANCE}

\section{A. Semiclassical scheme}

Now we generalize the Landauer approach of Ref. 9] developed for the electronic heat transport in superconductors with infinite transverse dimensions to mesoscopic samples and derive the along-the-vortex heat current. A general expression for the energy current along $z$ is

$$
\begin{aligned}
I_{\mathcal{E}}= & \int d^{2} r \sum_{\mu} \int \frac{d k_{z}}{\pi m}\left[\varepsilon_{\mu} u_{\mu k_{z}}^{*}\left(\hbar k_{z}-\frac{e}{c} A_{z}\right) u_{\mu k_{z}} n\left(\varepsilon_{\mu}\right)\right. \\
& \left.-\varepsilon_{\mu} v_{\mu k_{z}}^{*}\left(\hbar k_{z}+\frac{e}{c} A_{z}\right) v_{\mu k_{z}}\left[1-n\left(-\varepsilon_{\mu}\right)\right]\right] . \quad(25)
\end{aligned}
$$

Particles with the distribution $n(\varepsilon)$ carry the energy $+\varepsilon$ while the holes with the distribution $1-n(-\varepsilon)$ carry the energy $-\varepsilon$. If the electrodes are in equilibrium, $1-$ $n(-\varepsilon)=n(\varepsilon)$ at each electrode. The sum is taken over the states with $\varepsilon_{\mu}>0$. Using the BdG equations one can derive the important identity for the wave functions of the bound states $u_{\mu k_{z}}, v_{\mu k_{z}}$ belonging to the eigenvalues $\varepsilon_{\mu}$ of a given momentum $k_{z}$ along the $z$-axis ${ }^{9}$

$$
\begin{array}{r}
\int\left[u_{\mu k_{z}}^{*}\left(k_{z}-\frac{e}{\hbar c} A_{z}\right) u_{\mu k_{z}}-v_{\mu k_{z}}^{*}\left(k_{z}+\frac{e}{\hbar c} A_{z}\right) v_{\mu k_{z}}\right] d^{2} r \\
=\frac{m}{\hbar^{2}} \frac{\partial \varepsilon_{\mu}}{\partial k_{z}} .
\end{array}
$$

For the CdGM case this identity reveals a huge cancellation in its left hand side where each term is by the factor $k_{F} \xi$ larger than the right hand side. Within the 
quasiclassical approximation the l.h.s. vanishes for an infinite vortex line as a direct consequence of an approximate electron-hole symmetry. Note that such a cancellation does not occur in a finite-thickness slab $\frac{9}{9}$ where the CdGM states are not truly localized. Making use of this identity reduces the expression for the heat current to

$$
I_{\mathcal{E}}=\sum_{\mu} \int_{-k_{F}}^{+k_{F}} \varepsilon_{\mu} n\left(\varepsilon_{\mu}\right) \frac{\partial \varepsilon_{\mu}}{\partial k_{z}} \frac{d k_{z}}{\pi \hbar} .
$$

Equation (26) shows that, in a contrast to the electrical current, the energy flow is determined by the group velocity of excitations and this is why the conductance in Eq. (11) is much smaller than $\kappa_{\mathrm{Sh}}=\kappa_{0} N_{\mathrm{Sh}}$, where $\kappa_{0}=\pi T /(3 \hbar)$ is the heat conductance quantum defined in the Introduction.

The quasiparticles in the injector lead (left lead) having energies coinciding with the core-state levels penetrate into the core and are then transmitted through the vortex to the right lead with a probability $P_{\mu, k_{z}}$. In the same manner as it was done for the charge transport in mesoscopic conductors $\frac{10,11.21}{}$ we conclude that excitations with a positive group velocity $v_{g}=\partial \varepsilon_{\mu} / \hbar \partial k_{z}$ have the distribution $n_{L} P_{\mu, k_{z}}$ where $n_{L}$ is the distribution they had in the left lead. Similarly, excitations with a negative group velocity possess the distribution $n_{R} P_{\mu, k_{z}}$ where $n_{R}$ is their distribution in the right lead. Since $\varepsilon_{\mu}$ is an even function of $k_{z}$ we obtain

$$
I_{\mathcal{E}}=\sum_{\mu} \int_{0}^{k_{F}} \varepsilon_{\mu}\left[n_{L}\left(\varepsilon_{\mu}\right)-n_{R}\left(\varepsilon_{\mu}\right)\right] P_{\mu, k_{z}}\left|\frac{\partial \varepsilon_{\mu}}{\partial k_{z}}\right| \frac{d k_{z}}{\pi \hbar} .
$$

Defining the heat conductance as

$$
I_{\mathcal{E}}=\kappa\left(T_{L}-T_{R}\right)
$$

where $T_{L, R}$ are the temperatures of the left and right electrodes respectively, one finds

$$
\kappa=-\frac{1}{\pi T \hbar} \sum_{\mu} \int_{0}^{k_{F}} \varepsilon_{\mu}^{2} \frac{d n\left(\varepsilon_{\mu}\right)}{d \varepsilon_{\mu}} P_{\mu, k_{z}}\left|\frac{\partial \varepsilon_{\mu}}{\partial k_{z}}\right| d k_{z} .
$$

We assume that $T_{L}-T_{R} \ll T_{L, R} \approx T$. Using the conductance quantum $\kappa_{0}$, one can introduce the effective number of transverse modes propagating along the vortex line $N_{\text {eff }}=\kappa / \kappa_{0}$. Later in this section we neglect the scattering of excitations at the interface between the leads and the superconductor assuming that they are transmitted freely through the vortex, i.e. with $P_{\mu, k_{z}}=1$, deferring the effects of scattering till Section IIIB

For an infinite sample $R \gg R_{c}$ in the limit $\omega_{0} \ll T \ll$ $\Delta_{0}$ the sum in Eq. (28) can be replaced with the integral over $d \mu$ for the states with positive energies. Since $d n / d \varepsilon=-(4 T)^{-1} \cosh ^{-2}(\varepsilon / 2 T)$ we find

$$
\kappa=\frac{9 \zeta(3)}{2 \pi} \frac{T^{2}}{\hbar \omega_{0}} .
$$

Here $\zeta(n)$ is the Riemann zeta-function. Therefore

$$
N_{e f f}^{(\infty)}(T)=\frac{27 \zeta(3)}{2 \pi^{2}} \frac{T}{\omega_{0}} \sim k_{F} \xi \frac{T}{T_{c}},
$$

which coincides with the estimate discussed in the introduction (see Eq. (1)). At extremely low temperatures, $T \ll \omega_{0}$ :

$$
N_{e f f}^{(\infty)}(T)=\frac{3}{4 \pi^{2}}\left(\frac{\omega_{0}}{T}\right)^{2} \exp \left(-\frac{\omega_{0}}{2 T}\right) .
$$
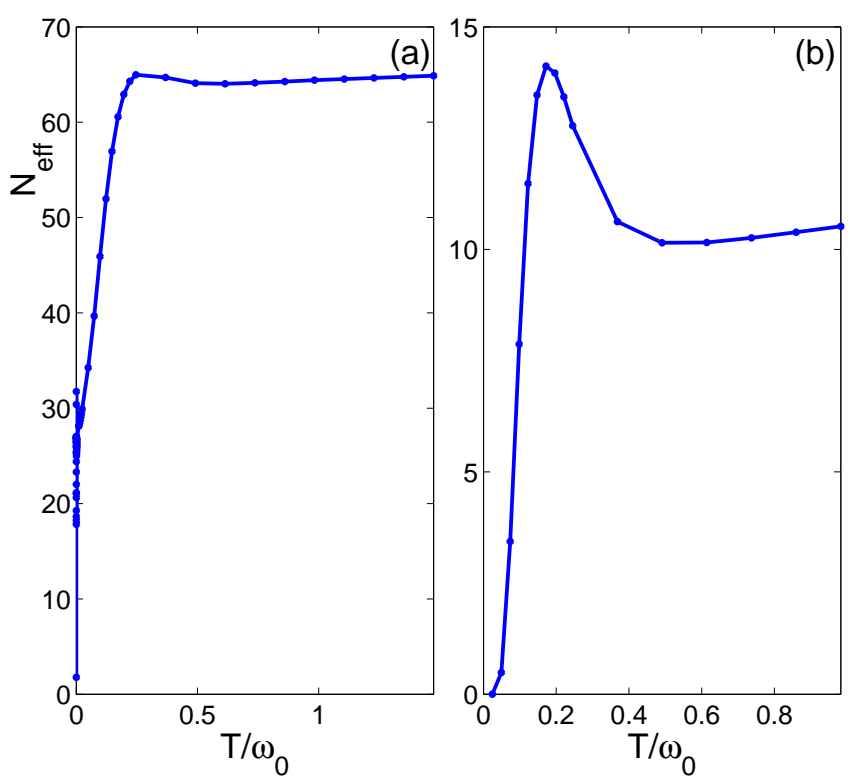

FIG. 2: The temperature dependence of the effective number of modes $N_{\text {eff }}(T)$ calculated using Eq. 28 with $P_{\mu, k_{z}}=1$ for two cylinders with $R / \xi=3.5$ (a) and $R / \xi=4.5$ (b). The gap is approximated by Eq. (20) with $\xi_{v}=\xi, k_{F} \xi=200$.

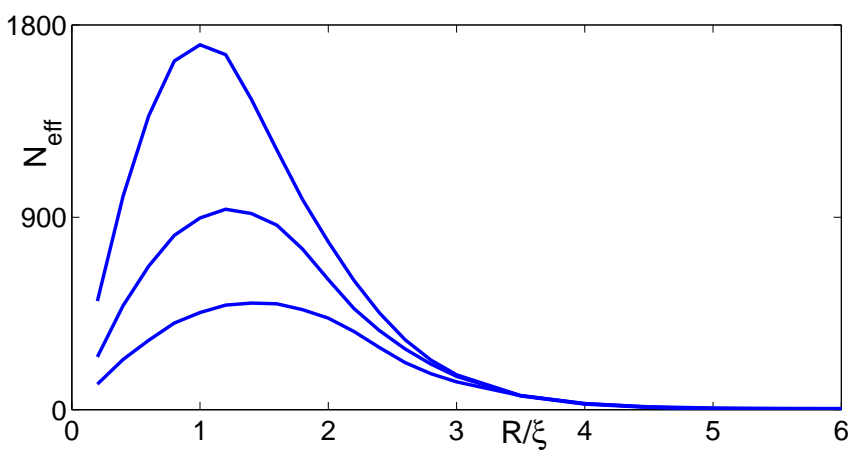

FIG. 3: The effective number of modes $N_{\text {eff }}$ according to Eq. (28) with $P_{\mu, k_{z}}=1$ as a function of the cylinder radius for various temperatures $T / \omega_{0}=0.3,1.0,3.0$ (from bottom to top). The gap is approximated by Eq. (20) with $\xi_{v}=\xi$, $k_{F} \xi=200$.

In a mesoscopic cylinder with $R \lesssim R_{c}$ there appear new conducting modes due to the oscillatory behavior of 
the energy levels. Indeed, since it is the absolute value of the group velocity that enters Eq. (28), both positive and negative slopes of the oscillating energy $\varepsilon_{\mu}\left(k_{z}\right)$ as a function of $k_{z}$ in Eq. (19) or in Fig. 1 contribute equally to the heat flux. This contribution is finite even for very low temperatures and is determined by the number of crossings of the constant energy line $\varepsilon \sim T$ by the oscillating spectrum. Thus this number represents the number of conducting modes $N_{e f f}^{(R)} \sim N_{k_{z}} N_{\mu}$, where $N_{k_{z}}$ is the number of crossings, as a function of $k_{z}$, of a spectral branch with a given angular momentum $\mu$, and $N_{\mu}$ is the number of the angular momentum branches that cross this line of energy $\varepsilon \sim T$. For large number of modes, i.e., when the oscillation amplitude is larger than $\omega_{0}$ we have ${ }^{16} N_{k_{z}} \sim k_{F} R\left(1-R / R_{c}\right)$ and $N_{\mu} \sim\left(2 \Delta_{0} / \omega_{0}\right) e^{-2 R / \xi}$. Finally, we get

$$
N_{e f f}^{(R)} \sim k_{F} R\left(1-R / R_{c}\right)\left(2 \Delta_{0} / \omega_{0}\right) e^{-2 R / \xi} .
$$

If $R \sim \xi$, then $N_{\text {eff }}^{(R)} \sim N_{\mathrm{Sh}} \sim\left(k_{F} \xi\right)^{2}$ which coincides with the estimate of the number of transport modes for a normal metal cylinder. We see therefore that the oscillations of the vortex-core energy spectrum in a mesoscopic cylinder lead to a drastic increase in the number of the conducting modes and, accordingly, to an enhanced electron thermal conductance through the vortex cores as compared to that in an infinite sample.

The temperature dependence of the effective number of modes calculated using Eq. (28) is shown in Fig. 2 The effective number of modes for $T \gg \omega_{0}$ is

$$
N_{e f f}^{(R)}(T) \simeq N^{\star}+\frac{27 \zeta(3)}{2 \pi^{2}} \frac{T}{\omega_{0}} .
$$

Here $N^{\star}$ is constant as function of temperature; it is small for $R>R_{c}$. However $N^{\star}$ grows and approaches the value $N_{\text {eff }}^{(R)}$ in Eq. (30) if the cylinder radius decreases below $R_{c}$ and the amplitude of oscillation increases. This asymptotic temperature dependence is observed already for $T \gtrsim 0.5 \omega_{0}$. The initial steep increase in $N_{e f f}$ in Fig. [2] is associated with the crossover into the regime of large number of modes when temperature is increased up to $T \sim \omega_{0}$. The constant value $N^{\star}$ leads to a linear temperature dependence of the heat conductance $\kappa(T)$ in the range $T \gtrsim \omega_{0}$. The linear in $T$ contribution can exceed the quadratic term given by Eq. (11) or by the second term in Eq. (31).

The effective number of modes calculated using Eq. (28) is shown in Fig. 3 as a function of the cylinder radius. Its non-monotonic dependence on $R$ agrees reasonably well with our simple estimate in Eq. (30).

\section{B. Exact quantum-mechanical analysis}

A disadvantage of the above semiclassical picture is that it does not provide a systematic way how to calculate the probability for excitations to penetrate into the vortex core modes, which is accompanied by reflection of excitations from the vortex ends at the boundaries between normal reservoirs and the mesoscopic superconductor. In this Section we derive the general quantummechanical expression for the heat conductance in superconductors directly from Eq. (25) and demonstrate that it has the form of the Landauer formula with the proper account of the scattering processes. Next we find the exact solution of the $\mathrm{BdG}$ equations and calculate the vortex heat conductance numerically.

We consider a cylindrical superconducting slab of the thickness $d$ (Fig. 4) confined between the two normal leads (reservoirs). The normal-superconducting interface is assumed ideally transparent. The axis of a trapped vortex line coincides with the superconducting cylinder axis. To calculate the heat conductance we solve the scattering problem for electron-like $(U)$ and hole-like $(V)$ quasiparticle wave functions within the BdG theory. It

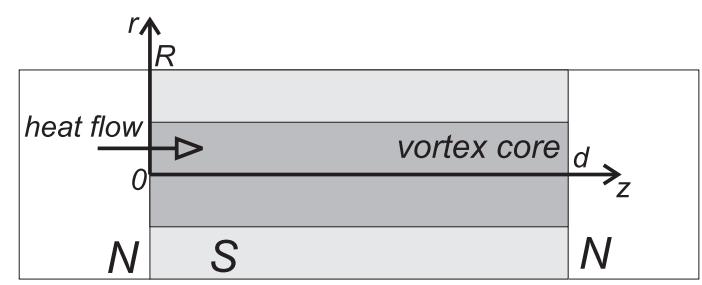

FIG. 4: Heat transport through a vortex line.

is convenient to consider four types of linearly independent states: (i) the state $\left(U_{1}, V_{1}\right)$ with an electron wave incident on a superconductor from the left lead in the absence of other incident electrons or holes; (ii) the state $\left(U_{2}, V_{2}\right)$ with a hole wave incident on a superconductor from the left lead; (iii) the state $\left(U_{3}, V_{3}\right)$ with an electron wave incident on a superconductor from the right lead; and (iv) the state $\left(U_{4}, V_{4}\right)$ with a hole wave incident on a superconductor from the right lead.

In the left lead, the first (electron) state has the form:

$$
\begin{aligned}
U_{1}= & \frac{e^{i(\mu+1 / 2) \theta}}{\sqrt{2 \pi}} g_{u, \mu s}(r) e^{+i k_{z} z} \\
& +\frac{e^{i(\mu+1 / 2) \theta}}{\sqrt{2 \pi}} \sum_{s^{\prime}} r_{u u, s s^{\prime}} g_{u, \mu s^{\prime}}(r) e^{-i k_{z u}^{\prime} z}, \\
V_{1}= & \frac{e^{i(\mu-1 / 2) \theta}}{\sqrt{2 \pi}} \sum_{s^{\prime}} r_{u v, s s^{\prime}} g_{v, \mu s^{\prime}}(r) e^{+i k_{z v}^{\prime} z}
\end{aligned}
$$

Here $g_{u, \mu s}(r)$ and $g_{v, \mu s}(r)$ are the transverse electron and hole modes of a normal metal wire with an angular momentum $\mu$ and a radial quantum number $s$ respectively. The transverse modes for each $\mu$ satisfy the boundary conditions $g_{u, \mu s}(R)=g_{v, \mu s}(R)=0$; they are normalized,

$$
\int_{0}^{R} g_{u, \mu s} g_{u, \mu s^{\prime}}^{*} r d r=\int_{0}^{R} g_{v, \mu s} g_{v, \mu s^{\prime}}^{*} r d r=\delta_{s, s^{\prime}}
$$


and have following form:

$$
\begin{aligned}
& g_{u, \mu s}= \frac{\sqrt{2} J_{|\mu+1 / 2|}\left(j_{s}^{(|\mu+1 / 2|)} r / R\right)}{R J_{|\mu+1 / 2|+1}\left(j_{s}^{(|\mu+1 / 2|)}\right)} ; \\
& g_{v, \mu s}=\frac{\sqrt{2} J_{|\mu-1 / 2|}\left(j_{s}^{(|\mu-1 / 2|)} r / R\right)}{R J_{|\mu-1 / 2|+1}\left(j_{s}^{(|\mu-1 / 2|)}\right)} .
\end{aligned}
$$

Here $J_{n}$ is the Bessel function of $n$-th order, $j_{s}^{(n)}$ is the $s$-th root of the equation $J_{n}\left(j_{s}^{(n)}\right)=0$. The solution Eqs. (32), (33) corresponds to the energy

$$
\varepsilon\left(\mu, s, k_{z}\right)=\frac{\hbar^{2}}{2 m}\left[k_{z}^{2}+\left(j_{s}^{(|\mu+1 / 2|)} / R\right)^{2}-k_{F}^{2}\right] .
$$

The wave numbers of the scattered electron and hole waves are $k_{z, u v}^{\prime} \equiv k_{z, u v}^{\prime}\left(\mu, s^{\prime}, k_{z}\right)$, where

$$
k_{z u, v}^{\prime}\left(\mu, s^{\prime}, k_{z}\right)=\sqrt{k_{F}^{2}-\left(j_{s^{\prime}}^{(|\mu \pm 1 / 2|)} / R\right)^{2} \pm 2 m \varepsilon / \hbar^{2}} .
$$

The factors $r_{u u, s s^{\prime}}$ and $r_{u v, s s^{\prime}}$ are the matrices of normal and Andreev reflection amplitudes for the processes with large and small transfers of longitudinal momentum, respectively. They are non-diagonal in $s$ and $s^{\prime}$ which accounts for scattering with a small change in the transverse momentum caused by an inhomogeneous vortex order parameter profile in the radial direction. The change in the radial momentum at a given energy $\varepsilon$ leads to a change in the longitudinal momentum such that the scattered waves have momenta $k_{z u}$ and $k_{z v}$ slightly different from the momentum $k_{z}$ in the incident wave.

Similarly, the state with an incident hole wave is

$$
\begin{aligned}
U_{2}= & \frac{e^{i(\mu+1 / 2) \theta}}{\sqrt{2 \pi}} \sum_{s^{\prime}} r_{v u, s s^{\prime}} g_{u, \mu s^{\prime}}(r) e^{-i k_{z u}^{\prime} z}, \\
V_{2}= & \frac{e^{i(\mu-1 / 2) \theta}}{\sqrt{2 \pi}} g_{v, \mu s}(r) e^{-i k_{z} z} \\
& +\frac{e^{i(\mu-1 / 2) \theta}}{\sqrt{2 \pi}} \sum_{s^{\prime}} r_{v v, s s^{\prime}} g_{v, \mu s^{\prime}}(r) e^{+i k_{z v}^{\prime} z} .
\end{aligned}
$$

The energy of the incident hole is

$$
\varepsilon\left(\mu, s, k_{z}\right)=-\frac{\hbar^{2}}{2 m}\left[k_{z}^{2}+\left(j_{s}^{(|\mu-1 / 2|)} / R\right)^{2}-k_{F}^{2}\right] .
$$

The transmitted state (iii) in the left lead is

$$
\begin{aligned}
U_{3} & =\frac{e^{i(\mu+1 / 2) \theta}}{\sqrt{2 \pi}} \sum_{s^{\prime}} t_{u u, s s^{\prime}} g_{u, \mu s^{\prime}}(r) e^{-i k_{z u}^{\prime} z}, \\
V_{3} & =\frac{e^{i(\mu-1 / 2) \theta}}{\sqrt{2 \pi}} \sum_{s^{\prime}} t_{u v, s s^{\prime}} g_{v, \mu s^{\prime}}(r) e^{+i k_{z v}^{\prime} z} .
\end{aligned}
$$

The waves $U_{4}, V_{4}$ in the state (iv) have the same form with the replacements $t_{u u, s s^{\prime}} \rightarrow t_{v u, s s^{\prime}}$ and $t_{u v, s s^{\prime}} \rightarrow$ $t_{v v, s s^{\prime}}$. The factors $t_{u u, s s^{\prime}}$ and $t_{v v, s s^{\prime}}$ describe the particle-particle and hole-hole transmission processes with a small change in the longitudinal momentum while the factors $t_{u v, s s^{\prime}}$ and $t_{v u, s s^{\prime}}$ describe the transmission with a large transfer of the longitudinal momentum involving normal scattering processes.

BdG equations (2) conserve the quasiparticle current

$$
\mathbf{J}_{q p}=\frac{1}{2 m}\left[u^{*}\left(-i \hbar \nabla-\frac{e}{c} \mathbf{A}\right) u-v^{*}\left(-i \hbar \nabla+\frac{e}{c} \mathbf{A}\right) v+c . c .\right] .
$$

For the incident and transmitted waves Eqs. (32), (33), etc., this conservation law yields the sum rules

$$
\begin{aligned}
& \sum_{s^{\prime}}\left(k_{z u}^{\prime}\left|r_{u u, s s^{\prime}}\right|^{2}+k_{z v}^{\prime}\left|r_{u v, s s^{\prime}}\right|^{2}\right. \\
& \left.+k_{z u}^{\prime}\left|t_{u u, s s^{\prime}}\right|^{2}+k_{z v}^{\prime}\left|t_{u v, s s^{\prime}}\right|^{2}\right)=k_{z}, \\
& \sum_{s^{\prime}}\left(k_{z v}^{\prime}\left|r_{v v, s s^{\prime}}\right|^{2}+k_{z u}^{\prime}\left|r_{v u, s s^{\prime}}\right|^{2}\right. \\
& \left.+k_{z v}^{\prime}\left|t_{v v, s s^{\prime}}\right|^{2}+k_{z u}^{\prime}\left|t_{v u, s s^{\prime}}\right|^{2}\right)=k_{z} .
\end{aligned}
$$

In the quasiclassical approximation, the transverse momentum is conserved, therefore

$$
r_{u v, s s^{\prime}}=r_{u v, s} \delta_{s, s^{\prime}}, r_{v u, s s^{\prime}}=r_{v u, s} \delta_{s, s^{\prime}} .
$$

At the same time, the processes with large longitudinal momentum transfer are prohibited

$$
r_{u u, s s^{\prime}}=r_{v v, s s^{\prime}}=t_{u v, s s^{\prime}}=t_{v u, s s^{\prime}}=0 .
$$

However, in the exact BdG formalism used in our numerical calculations, we keep the full set of probabilities including $r_{u u, s s^{\prime}}, r_{v v, s s^{\prime}}, t_{u v, s s^{\prime}}$, and $t_{v u, s s^{\prime}}$.

The heat flow through the cylinder cross section in the left lead can be calculated summing up the states (i)-(iv):

$$
\begin{aligned}
I_{\mathcal{E}}= & \sum_{\mu, s} \int \frac{d k_{z}}{2 \pi} \int r d r d \theta \frac{\hbar}{m}\left\{\left[\varepsilon \cdot \operatorname{Im}\left\{U_{1}^{*} \nabla U_{1}\right\} n_{L}(\varepsilon)-\varepsilon \cdot \operatorname{Im}\left\{V_{1}^{*} \nabla V_{1}\right\} \cdot\left(1-n_{L}(-\varepsilon)\right)\right]\right. \\
& +\left[\varepsilon \cdot \operatorname{Im}\left\{U_{2}^{*} \nabla U_{2}\right\} n_{L}(\varepsilon)-\varepsilon \cdot \operatorname{Im}\left\{V_{2}^{*} \nabla V_{2}\right\} \cdot\left(1-n_{L}(-\varepsilon)\right)\right] \\
& +\left[\varepsilon \cdot \operatorname{Im}\left\{U_{3}^{*} \nabla U_{3}\right\} n_{R}(\varepsilon)-\varepsilon \cdot \operatorname{Im}\left\{V_{3}^{*} \nabla V_{3}\right\} \cdot\left(1-n_{R}(-\varepsilon)\right)\right] \\
& \left.+\left[\varepsilon \cdot \operatorname{Im}\left\{U_{4}^{*} \nabla U_{4}\right\} n_{R}(\varepsilon)-\varepsilon \cdot \operatorname{Im}\left\{V_{4}^{*} \nabla V_{4}\right\} \cdot\left(1-n_{R}(-\varepsilon)\right)\right]\right\} .
\end{aligned}
$$


The sum is taken over the propagating quasiparticle states with $\operatorname{Im} k_{z}=0$ and $\varepsilon \geq 0$ (we thus take into account both incident electrons and incident holes).

Using the sum rules Eqs. (41), (42) and transforming to the energy integral, the heat current becomes

$$
I_{\mathcal{E}}=\frac{1}{\pi \hbar} \int_{0}^{\infty} \varepsilon\left[n_{L}(\varepsilon)-n_{R}(\varepsilon)\right] \mathcal{T}(\varepsilon) d \varepsilon,
$$

where the transfer factor

$$
\mathcal{T}(\varepsilon)=\sum_{\mu, s, s^{\prime}}\left[\left|t_{u u, s s^{\prime}}\right|^{2}+\left|t_{u v, s s^{\prime}}\right|^{2}+\left|t_{v u, s s^{\prime}}\right|^{2}+\left|t_{v v, s s^{\prime}}\right|^{2}\right]
$$

is the sum of partial transmission probabilities. Finally, the dimensionless heat conductance (the effective number of modes) becomes

$$
N_{\text {eff }}=\frac{\kappa}{\kappa_{0}}=-\frac{3}{\pi^{2} T^{2}} \int_{0}^{\infty} \varepsilon^{2} \frac{d n(\varepsilon)}{d \varepsilon} \mathcal{T}(\varepsilon) d \varepsilon .
$$

Equations (44), (45), (46) are the exact quantummechanical expressions for the heat conductance. They correspond to the Landauer formula ${ }^{10}$ in which the transmission probabilities are defined through the full scattering problem for excitations in superconductors.

The semiclassical approach developed in the previous section assumes that each particle or hole, incident on one of the superconductor/normal-metal interfaces, with the energy and momentum corresponding to the one or another propagating vortex core mode is subsequently transmitted along this channel with a probability $P_{i}$ to the second normal lead:

$$
\left|t_{u u, s}\right|^{2}=\sum_{s_{i}^{+}} P_{i} \delta_{s, s_{i}^{+}}
$$

provided the direction of the quasiparticle momentum along $z$ coincides with the direction of the group velocity in the vortex core, and

$$
\left|t_{v v, s}\right|^{2}=\sum_{s_{i}^{-}} P_{i} \delta_{s, s_{i}^{-}}
$$

in case where the direction of the quasiparticle momentum along $z$ is opposite to the direction of the group velocity. The index $i$ denotes the set of quantum numbers $\mu, k_{z}$; the sum is taken over such quantum numbers $s_{i}^{+}$ and $s_{i}^{-}$which satisfy the energy conservation equations $\varepsilon_{\mu}\left[k_{z}\left(s_{i}^{ \pm}\right)\right]=\varepsilon$ for increasing or decreasing branches of $\varepsilon_{\mu}$ as functions of $k_{z}$, respectively (we emphasize that the quantum number $s$ is regarded as continuous in the semiclassical approximation). Using $t_{u v}=t_{v u}=0$ one can write Eq. (45) as

$$
\mathcal{T}(\varepsilon)=\sum_{\mu} \int_{0}^{k_{F}} P_{\mu, k_{z}}\left|\frac{\partial \varepsilon_{\mu}}{\partial k_{z}}\right| \delta\left(\varepsilon-\varepsilon_{\mu}\left(k_{z}\right)\right) d k_{z} .
$$

As a result, Eq. (44) coincides with Eq. 27). Under the assumption of complete transmission $P_{\mu, k_{z}}=1$ the transfer factor $\mathcal{T}(\varepsilon)$ is simply equal to the number of quasiparticle modes propagating along the vortex core for a given energy $\varepsilon$.
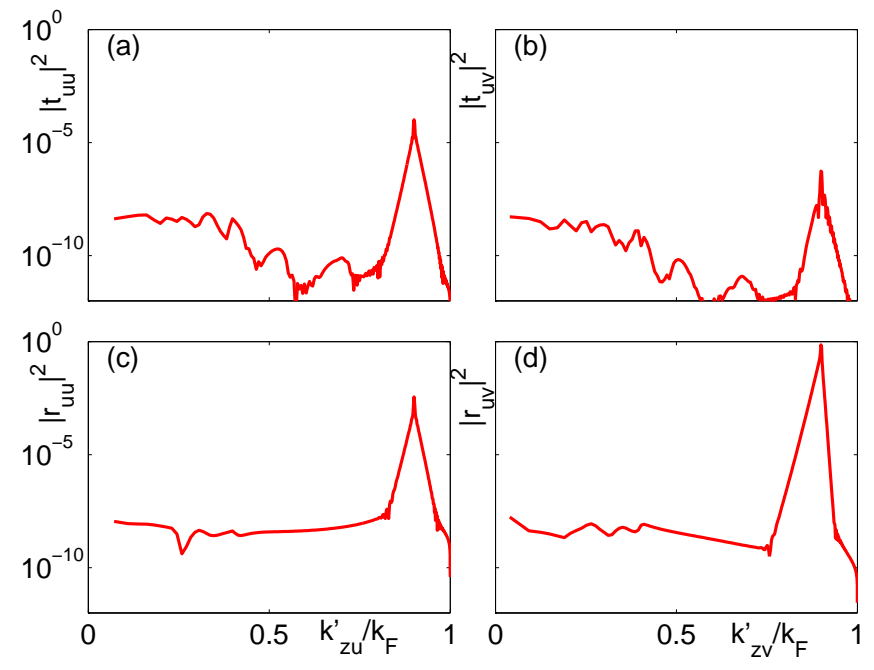

FIG. 5: (a), (b) The transmission $\left|t_{u u}\right|^{2},\left|t_{u v}\right|^{2}$ and (c), (d) reflection $\left|r_{u u}\right|^{2},\left|r_{u v}\right|^{2}$ probabilities, respectively, as functions of momenta $k_{z u}^{\prime}$ and $k_{z v}^{\prime}$ of scattered waves for the incident electron-like quasiparticle wave with $\mu=-1 / 2, k_{z} / k_{F}=0.9$, and $\varepsilon=0.49 \omega_{0}$. Parameters are: $R=4.5 \xi, d / \xi=16$.

\section{Numerical results}

Here we present the results of numerical calculations of the vortex heat conductance for a typical parameter $\Delta_{0} / E_{F}=0.01\left(k_{F} \xi=200\right)$. The vortex core was approximated by Eq. (20) with $\xi_{v}=\xi$. The critical radius estimated by Eq. (22) for the chosen set of parameters is $R_{c} \simeq 3.96 \xi$. The calculations were carried out for two values of $R=3.5 \xi<R_{c}$ and $R=4.5 \xi>R_{c}$, and for several values of superconductor lengths $d / \xi$ varied from 16 up to 2048. The corresponding energy spectra for propagating quasiparticle waves in a cylinder of an infinite length tabulated using Eq. (21) are shown in Fig. 10

We calculate the scattering matrix of the normal modes incident on the mesoscopic superconductor with a trapped vortex line using a representation of the BdG operator in the truncated basis of the normal-metal eigenfunctions. We solve the system of linear equations which correspond to the boundary conditions at the normalmetal/superconductor interfaces and to the conditions at the infinity (radiation condition). Typical scattering matrix coefficients as functions of the momentum of scat-

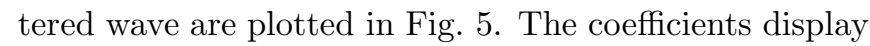
peaks around $k_{z u, v}=k_{z}$. The energy dependence of the transfer factor $\mathcal{T}(\varepsilon)$ calculated using Eq. 45) for two lengths $d$ of the vortex line is shown in Figs. [6] The linear in $\varepsilon / \omega_{0}$ term in the transfer factor corresponds to the increasing number of CdGM modes with $|\mu| \omega_{0} \leq \varepsilon$ which contribute to the heat transport.

The temperature dependence of the heat conductance calculated using Eq. (46) and plotted in Fig. [7 is qualitatively similar to that obtained on the basis of the semiclassical model (see Fig. 21). In particular, the slope of the linear asymptotic dependence $\left(T>\omega_{0}\right)$ of $N_{\text {eff }}$ vs 

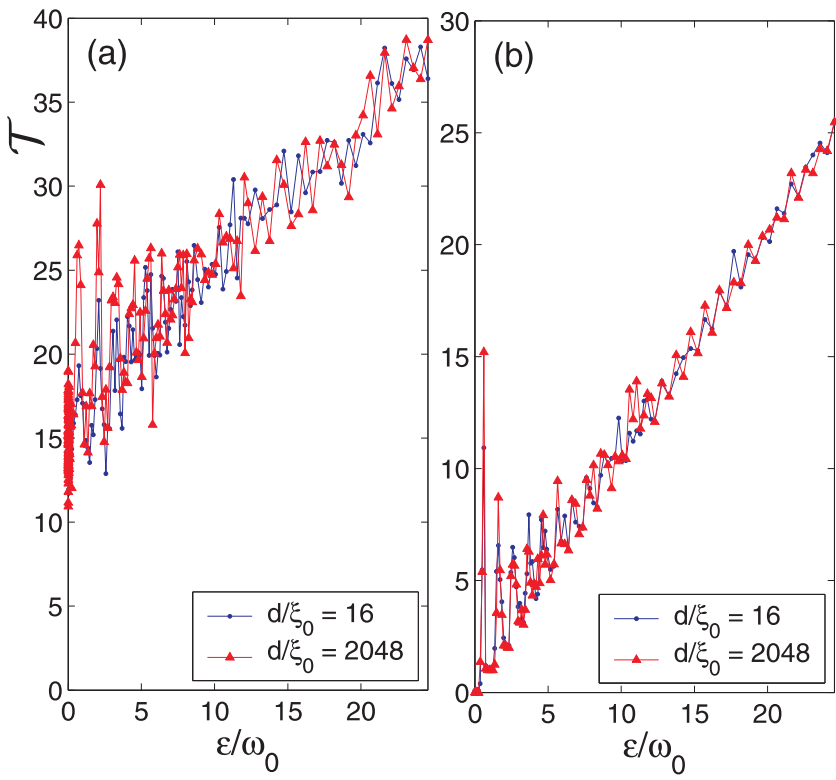

FIG. 6: (Color online) The transfer factor $\mathcal{T}(\varepsilon)$ for different vortex line lengths $d$ (blue line is for $d / \xi=16$ and red line is for $d / \xi=2048$ ) in the cylinder with $R / \xi=3.5$ (a) and $R / \xi=4.5(\mathrm{~b})$.

temperature is indeed well described by the analytical approach. However, the value $N_{\text {eff }}$ calculated numerically is about seven times smaller than that evaluated within the semiclassical approach with $P_{\mu, k_{z}}=1$. Such deviation is obviously caused by a finite probability for excitations to penetrate into the vortex core states at the normal-lead/superconductor interfaces. The conductance peak at $T^{*} \simeq 0.2 \omega_{0}$ which is most pronounced for the radius $R>R_{c}$ (see Fig. $7 \mathrm{~b}$ ) is associated with the contribution of the lowest oscillating CdGM level to the transfer factor $\mathcal{T}(\varepsilon)$.

In our numerical calculations we do not observe the power-law dependence of the thermal conductance on the thickness $d$ predicted in Ref. [9] which is associated with the ballistic quasiparticle trajectories passing close to the vortex axis. This power-law behavior is expected to dominate only for much shorter $d$; the transport associated with such trajectories appears to be closed already for our set of material parameters and $d>16 \xi$ since it would result in $N_{\text {eff }} \lesssim 10^{-3}$.

\section{VORTEX IN A CYLINDER WITH A ROUGH SURFACE}

In the previous sections we calculated the electronic spectrum and the heat transport for a sample with a perfect surface. One can expect, however, that surface imperfections and roughness would reduce the amplitude of spectrum oscillations. Making use of the approach developed in Section 1 we analyze here how surface roughness
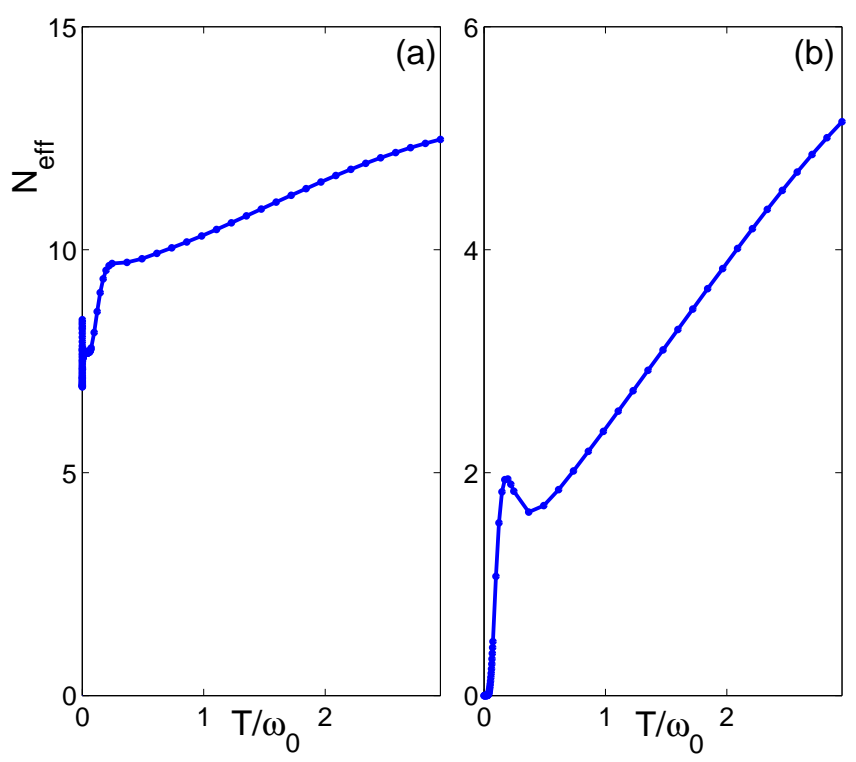

FIG. 7: The effective number of modes $N_{\text {eff }}(T)$ calculated numerically as a function of temperature for the vortex line length $d / \xi_{0}=16$ in cylinders with $R / \xi_{0}=3.5$ (a) and $R / \xi_{0}=$ 4.5 (b).

affects the vortex-core spectrum and the heat transport mediated by the vortex core states. We show that there exists a broad class of surface imperfections which do not destroy mesoscopic spectrum oscillations completely; in this case the heat transport through vortices still remains significantly enhanced as compared to that in the bulk system.

Let us treat the potential $\hat{V}$ in Eq. (16) as a perturbation assuming the cylinder radius to be sufficiently large. The solution to this equation within the zero-order in $\hat{V}$ is a set of orthogonal and normalized eigenfunctions Eq. (18). The first-order correction to the energy levels $\delta \varepsilon \equiv \varepsilon-\epsilon(\mu)$ is

$$
\delta \varepsilon=\frac{\Delta_{0}}{\pi \Lambda} \int_{0}^{2 \pi} e^{-D_{\theta}} \cos \left[\alpha_{\theta}-\pi \mu+\pi / 2\right] d \theta .
$$

Consider several examples. Assume first that the cylinder has a smooth surface $R(\theta)$. The main contribution to the integral in Eq. (47) comes then from the stationary phase angles $\theta_{j}$ defined by the condition $d \alpha_{\theta} /\left.d \theta\right|_{\theta_{j}}=2 k_{\perp} d R /\left.d \theta\right|_{\theta_{j}}=0$. In the limit $k_{\perp} R^{\prime \prime} \gg 1$ we thus obtain

$$
\begin{aligned}
\delta \varepsilon= & \frac{\Delta_{0}}{\Lambda} \sum_{j} \sqrt{\frac{2}{\pi k_{\perp}\left|R^{\prime \prime}\left(\theta_{j}\right)\right|}} e^{-D_{\theta_{j}}} \\
& \times \cos \left[\alpha_{\theta_{j}}-\pi \mu+\pi / 2+\frac{\pi}{4} \operatorname{sign}\left(R^{\prime \prime}\left(\theta_{j}\right)\right)\right] .
\end{aligned}
$$

For a simple realization $R(\theta)=R_{0}+a \cos (m \theta)$, where $a \ll \xi$, the correction to the energy takes the form:

$$
\delta \varepsilon=\left(2 \Delta_{0} / \Lambda\right) e^{-D_{0}} \cos \left(\alpha_{0}-\pi \mu+\pi / 2\right) J_{0}\left(2 k_{\perp} a\right)
$$


where $\alpha_{0}=2 k_{\perp} R_{0}$ and $D_{0} \equiv D\left(R_{0}\right)$. In the limit $k_{\perp} a \gg$ 1 we obtain

$$
\begin{aligned}
\delta \varepsilon= & \left(2 \Delta_{0} / \Lambda\right)\left(\pi k_{\perp} a\right)^{-1 / 2} e^{-D_{0}} \\
& \times \cos \left(\alpha_{0}-\pi \mu+\pi / 2\right) \cos \left(2 k_{\perp} a-\pi / 4\right) .
\end{aligned}
$$

The amplitude of oscillations is decreased by a factor $\left(k_{F} a\right)^{-1 / 2} \ll 1$. Nevertheless, the amplitude $\delta \varepsilon \gg \omega_{0}$ as long as $e^{-D_{0}} \gg\left(k_{F} a\right)^{1 / 2}\left(k_{F} \xi\right)^{-1}$. Note that the r.h.s. of this inequality is a small parameter.

Let us now assume that the cylinder has a rough surface such that the radius is $R(\theta)=R_{0}+a(\theta)$, where $a(\theta)$ is a random function of sample realizations with a zero ensemble average $\langle a\rangle=0$ having a Gaussian distribution

$$
w_{1}(a)=\frac{1}{\sqrt{2 \pi} \sigma} \exp \left[-\frac{a^{2}}{2 \sigma^{2}}\right] \text {. }
$$

Here $\sigma \ll \xi$ is the mean square value: $\left\langle a^{2}\right\rangle=\sigma^{2}$. Averaging of Eq. (47) gives

$$
\begin{aligned}
\langle\delta \varepsilon\rangle & =\frac{\Delta_{0}}{\pi \Lambda} e^{-D_{0}} \cos \left(\alpha_{0}-\pi \mu+\pi / 2\right) \int_{0}^{2 \pi}\left\langle e^{2 i k_{\perp} a(\theta)}\right\rangle d \theta \\
& =\frac{2 \Delta_{0}}{\Lambda} e^{-D_{0}} \cos \left(\alpha_{0}-\pi \mu+\pi / 2\right) e^{-2 k_{\perp}^{2} \sigma^{2}} .
\end{aligned}
$$

This average vanishes for $k_{F} \sigma \gg 1$.

However, the energy level fluctuations are considerable. To prove this we calculate the mean square energy fluctuation assuming a Gaussian distribution for $a_{1}=a\left(\theta_{1}\right)$ and $a_{2}=a\left(\theta_{2}\right)$ :

$$
w_{2}\left(a_{1}, a_{2}\right)=\frac{1}{2 \pi \sigma^{2} \sqrt{1-K^{2}}} \exp \left[-\frac{r^{2}}{2\left(1-K^{2}\right) \sigma^{2}}\right]
$$

where $r=\sqrt{a_{1}^{2}+a_{2}^{2}-2 K a_{1} a_{2}}$ and $K=K\left(\theta_{1}-\theta_{2}\right)=$ $\left\langle a_{1} a_{2}\right\rangle / \sigma^{2}$ is the correlation coefficient. This correlation coefficient should be an even and $2 \pi$-periodic function of angle: $K(\theta)=K(-\theta)=K(\theta+2 \pi)$. For $\theta_{1}=\theta_{2}$ we get $K=1$. The correlation coefficient decays as we increase $\left|\theta_{1}-\theta_{2}\right|$ value from 0 to $\pi$. We obtain

$$
\begin{aligned}
\left\langle\delta \varepsilon^{2}\right\rangle & \simeq \frac{\Delta_{0}^{2}}{\pi^{2} \Lambda^{2}} e^{-2 D_{0}} \int_{0}^{2 \pi} \int_{0}^{2 \pi}\left\langle\cos \left(\alpha_{\theta_{1}}-\pi \mu+\pi / 2\right) \cos \left(\alpha_{\theta_{2}}-\pi \mu+\pi / 2\right)\right\rangle d \theta_{1} d \theta_{2} \\
& =\frac{\Delta_{0}^{2}}{2 \pi^{2} \Lambda^{2}} e^{-2 D_{0}} \int_{0}^{2 \pi} \int_{0}^{2 \pi}\left[\cos \left(2 \alpha_{0}\right)\left\langle e^{2 i k_{\perp}\left(a_{1}+a_{2}\right)}\right\rangle+\left\langle e^{2 i k_{\perp}\left(a_{1}-a_{2}\right)}\right\rangle\right] d \theta_{1} d \theta_{2} .
\end{aligned}
$$

For $k_{\perp} \sigma \gg 1$ the second term is only important. Using the characteristic function for a Gaussian process

$$
\left\langle e^{i\left(u_{1} a_{1}+u_{2} a_{2}\right)}\right\rangle=e^{-\frac{1}{2} \sigma^{2}\left(u_{1}^{2}+u_{2}^{2}+2 K u_{1} u_{2}\right)}
$$

where $u_{1}=-u_{2}=2 k_{\perp}$ we get

$$
\int_{0}^{2 \pi} \int_{0}^{2 \pi}\left\langle e^{2 i k_{\perp}\left(a_{1}-a_{2}\right)}\right\rangle d \theta_{1} d \theta_{2}=4 \pi \int_{0}^{\pi} e^{-4 \sigma k_{\perp}^{2}(1-K(\theta))} d \theta
$$

Expanding the correlation coefficient for small angles, $K(\theta) \simeq 1-\left|K_{\theta \theta}^{\prime \prime}(0)\right| \theta^{2} / 2$, and introducing the correlation radius $\ell=R_{0} / \sqrt{\left|K^{\prime \prime}(0)\right|}$ we find

$$
\left\langle\delta \varepsilon^{2}\right\rangle \simeq \frac{\Delta_{0}^{2} \ell}{\Lambda^{2} k_{\perp} \sigma R_{0}(2 \pi)^{1 / 2}} e^{-2 D_{0}} .
$$

This expression is valid if the range of angles contributing to the integral is small, $\theta \sim 1 / \sigma k_{\perp} \sqrt{\left|K^{\prime \prime}(0)\right|} \ll 1$. Taking $\ell \sim \sigma$ we obtain

$$
\sqrt{\left\langle\delta \varepsilon^{2}\right\rangle} \simeq \frac{\Delta_{0}}{\Lambda \sqrt{k_{\perp} R_{0}}(2 \pi)^{1 / 4}} e^{-D_{0}} \gg|\langle\delta \varepsilon\rangle| .
$$

We see that the mean square fluctuation is much larger than the CdGM interlevel spacing if the sample radius is not very large, $e^{-D_{0}} \gg\left(k_{F} R_{0}\right)^{1 / 2}\left(k_{F} \xi\right)^{-1}$. Note again that the r.h.s. here is a small parameter.

When the correlation radius increases and sample dimensions are small $R_{0}<\ell / k_{\perp} \sigma$ we can take $K \simeq 1$ for all $\theta$. As a result

$$
\sqrt{\left\langle\delta \varepsilon^{2}\right\rangle} \simeq\left(\Delta_{0} \sqrt{2} / \Lambda\right) e^{-D_{0}}
$$

In this limit the fluctuations of energy levels are universal in the sense that they do not depend on $\ell$ and $\sigma$, though do depend on the sample size.

To summarize, we see that imperfections on sample surface do not always remove the spectrum oscillations. We have demonstrated that the number of propagating 
quasiparticle modes responsible for the heat transport in a mesoscopic sample can still be essentially large for smooth surfaces with comparatively big variations in the radius and also for Gaussian imperfections with fluctuations as large as $\sigma \gg k_{F}^{-1}$.

\section{CONCLUSIONS}

We have investigated the electronic thermal transport along the flux line traversing a mesoscopic superconducting sample having the shape of a cylinder with the arbitrary cross section. We have shown that the normal quasiparticle scattering at the sample boundaries results in the essential increase in the number of modes propagating along the vortex core. We have found that the semiclassical version of the Landauer theory provides an adequate qualitative description of the heat transport carried by these modes. We have verified this conclusion by the detailed numerical analysis of the exact quantum-mechanical scattering problem described by the Bogoliubov-de Gennes theory. For small-radius cylinders, such that $R \lesssim R_{c}$, the minigap in the vortex spectrum is suppressed and the conductance becomes a linear function of temperature in the range $T \gtrsim \omega_{0}$. This linear term in the vortex conductance increases with the decrease in the cylinder radius; it can strongly exceed the quadratic term Eq. (1) which usually dominates in the bulk system at $\omega_{0} \ll T \ll T_{c}$.

We have analyzed how imperfections at the cylinder surface affect the quasiparticle spectrum. We have found that there exists a wide class of surface imperfections that give rise to only partial reduction in the number of the conducting modes mediating the heat conductance, even if the dimensions of imperfections exceed the atomic scale $k_{F}^{-1}$.

\section{Acknowledgments}

This work was supported in part by the US DOE Office of Science under contract No. W-31-109-ENG-38, by Russian Foundation for Basic Research, the Program "Quantum Macrophysics" of the Russian Academy of Sciences, Russian Science Support Foundation, and "Dynasty" Foundation. ASM acknowledges the support by the Academy of Finland.
1 C. Caroli and M. Cyrot, Phys. Kond. Materie 4, 285 (1965).

2 K. Maki, Phys. Rev. 158, 397 (1967).

3 J. Bardeen, R. Kümmel, A. E. Jacobs, and L. Tewordt, Phys. Rev. 187, 556 (1969).

4 J. Bardeen, G. Rickayzen, and L. Tewordt, Phys. Rev. 113, 982 (1959).

5 N. B. Kopnin, Zh. Eksp. Teor. Fiz. 69, 364 (1975) [Sov. Phys. JETP, 42, 186 (1975)].

6 J. Lowell and J. B. Sousa, J. Low Temp. Phys. 3, 65 (1970).

7 W. F. Vinen, E. M. Forgan, C. E. Gough, and M. J. Hood, Physica 55, 94 (1971).

8 C. Caroli, P. G. de Gennes, J. Matricon, Phys. Lett. 9, 307 (1964).

9 N. B. Kopnin, A. S. Mel'nikov, and V. M. Vinokur, Phys. Rev. B 68, 054528 (2003).

10 P. N. Butcher, J. Phys.: Condens. Matter 2, 4869 (1990).

11 H. van Houten, L. W. Molenkamp, C. W. J. Beenakker, and C. T. Foxon, Semicond. Sci. Technol. 7, B215 (1992).

12 L.G.C. Rego and G. Kirczenow, Phys. Rev. B 59, 13080 (1999).

13 O. Chiatti, J.T. Nicholls, Y.Y. Proskuryakov, N. Lumpkin,
I. Farrer, and D.A. Ritchie, Phys. Rev. Lett. 97, 056601 (2006).

14 F. Giazotto, T. T. Heikkilä, A. Luukanen, A. M. Savin, and J. P. Pekola, Rev. Mod. Phys. 78, 217 (2006).

15 G. Boato et al., Solid St. Commun. 3, 173 (1965); D. S. McLachlan, Solid St. Commun. 8, 1589 (1970); V. A. Schweigert et al., Phys. Rev. Lett. 81, 2783 (1998); A. K. Geim et al., Phys. Rev. Lett. 851528 (2000); L. F. Chibotaru et al., Nature 408, 833 (2000).

16 N. B. Kopnin, A. S. Mel'nikov, V. I. Pozdnyakova, D. A. Ryzhov, I. A. Shereshevskii, V. M. Vinokur, Phys. Rev. Lett. 95, 197002 (2005).

17 M. Titov, A. Ossipov, and C. W. J. Beenakker, cond-mat/0609623

18 A. A. Koulakov and A. I. Larkin, Phys. Rev. B 60, 14597 (1999).

19 Brun E. Hansen, Phys. Lett. A 27, 576 (1968).

20 A. S. Mel'nikov and V. M. Vinokur, Nature, 415, 60 (2002); Phys. Rev. B 65, 224514 (2002).

21 S. Datta, Electronic transport in mesoscopic systems (Cambridge 1995). 